Physics Vol. 2, No. 2, pp. 95-105, 1965. Physics Publishing Co. Printed in Great Britain.

\title{
IS A NON-RELATIVISTIC APPROXIMATION POSS IBLE FOR THE INTERNAL DYNAMICS OF "ELEMENTARY" PARTICLES? *
}

\author{
G. MORPURGO \\ Istituto di Fisica dell'Universita di Genova \\ Sezione di Genova dell'Istituto Nazionale di Fisica Nucleare, \\ Genova, Italy \\ (Received 28 April 1965)
}

\begin{abstract}
It is pointed out that, contrary to what it is usually implied, the internal dynamics in a compound model of an "elementary" particle is not necessarily relativistic. If the force which binds the constituent objects has a finite range and is not too singular, the relativistic character of the internal motion only depends on the range of such force and on the mass of the constituent particles, not on the depth of the potential well. For instance, for a quark antiquark model of the octet bosons with a quark mass of $5 \mathrm{GeV}$ and a range of the binding force $\left(5 m_{\pi}\right)^{-1}$, one has $(p / M)^{2}=1 / 40$, a non-relativistic situation quite similar to that occurring in nuclei.

Starting from this observation it is pointed out that several features of the $\mathrm{SU}_{3}$ and $\mathrm{SU}_{6}$ dynamics can be understood by writing a non-relativistic Hamiltonian for quarks, in a way similar to that which is used in writing an Hamiltonian for a nucleus. More precisely: (1) one can understand why the Gell Mann-Okubo $\mathrm{SU}_{3}$ mass formula for baryons works much better than its perturbative derivation would imply; (2) one can obtain the $-3 / 2$ ratio between the magnetic moments of proton and neutron, due to $\mathrm{SU}_{6}$, by a very simple calculation, which, at the same time exhibits how peculiar is the situation for the absolute values of the magnetic moments; some interesting aspects of the situation, though implied in the conventional derivation, are masked there under the algebra; (3) the point of view taken in this paper leads to the conclusion that $\mathrm{SU}_{6}$ should possibly be valid only at non-relativistic energies of the interacting particles.
\end{abstract}

\section{Introduction}

THE PURPOSE of the present note, which we hope to expand in future, is to draw attention to a circumstance which has been considered almost obvious since the work of Fermi and Yang [1] but which is, in our opinion, not evident at all.

* Paper presented at the Frascati meeting of the INFN on high energy physics-April 12, 1965. 
The question is whether, assuming a compound model for elementary particles, the motion of the constituent particles is a relativistic one, or a non-relativistic one like e.g. in positronium or in a nucleus.

It has almost always considered obvious or implied that the motion is a relativistic one, indeed an extremely relativistic one. The reason for this belief can probably be traced back to two circumstances. The first is that Fermi and Yang used, for their construction of the pion as a $N \bar{N}$ system, a potential well where the range of the well was assumed equal to the nucleon Compton wavelength $M_{N}{ }^{-1}$. Doing so the motion of the $N \bar{N}$ system is necessarily relativistic simply because of the indeterminacy principle; indeed, the indeterminacy in the momentum must be of the order $M_{N}$, so that we have that the order of magnitude of $\left(\Delta p / M_{N}\right)^{2}$ is $\sim 1$, that is the motion is fully relativistic. The second circumstance has been that with a long range force the average value of the kinetic energy has the same order of magnitude as the average value of the potential energy (virial theorem); so that since the potential energy has to be of the order of the rest energy of the particles which are bound, the kinetic energy has the same order of magnitude and the problem is clearly fully relativistic.

Now assume for a moment that the range of the force giving the attraction between a nucleon and an anti-nucleon to produce the pion is not $M_{N}{ }^{-1}$ but $\left(2 m_{\pi}\right)^{-1}$, that corresponding to two pion masses; that is we assume for a moment a strongly attractive potential well with range $\left(2 m_{\pi}\right)^{-1}$. It should be emphasized that this assumption is here made only to exemplify what we have in mind and may be in this case in contrast with the experimental facts on nucleon-antinucleon scattering and annihilation. In this case the indeterminacy in momentum is $\Delta p \simeq 2 m_{\pi}$ and the average kinetic energy is of the order of $\left(2 m_{\pi}\right)^{2} / M$. The ratio between kinetic energy and nucleon rest energy is $\left(2 m_{\pi}\right)^{2} / M_{N}{ }^{2} \sim 1 / 12$, a reasonably non-relativistic situation.

In other words we can have a situation in which the potential well which binds the nucleon and antinucleon is so deep as to cancel the masses of the particles which are bound; and has also a range much larger than the Compton wave length of the constituent particles. The relativistic or non-relativistic character of the situation for the ground state does not depend on the depth of the potential; it only depends on the range of the potential well and the mass of the constituent particles."

We have so far considered the Fermi-Yang model of the pion, but in reality these ideas were originated thinking of the quark structure of particles $[2,3,4]$. Think of the pion as a quarkantiquark compound and assume that quarks are real particles (not only mathematical structures) and that their mass is rather large, say $M=5 \mathrm{GeV}$; we do not know of course the exact value. The interaction between quarks can be transmitted by quark-antiquark compounds; to get attraction it can be transmitted e.g. by a particle of the vector octet. The range is therefore $\sim\left(5 m_{\pi}\right)^{-1}$ and the kinetic energy $T$ of the quark-antiquark compound is $\left(5 m_{\pi}\right)^{2} / M$. With $M=5 \mathrm{GeV}$ the ratio $T / M$ is $\sim 1 / 40$, a clearly non-relativistic situation quite similar to that which occurs in nuclei.

If this is so a number of apparently mysterious facts can be, at least qualitatively, explained.

We first consider a "simple" $S U_{3}$ theory $[5,6]$ and next discuss $S U_{6}$.

The first point which becomes clear is that if particles are composed of quarks, a description in which the number of quarks and antiquarks contained in a given particle is fixed is a very good description, in the same sense in which a description of a nucleus with a wave function with a fixed number of nucleons is a very good description. It is true that a nucleon- 
antinucleon pair may be present in a nucleus in addition to the nucleons which it contains, but in practice a good description of a nucleus is obtained neglecting such virtual pairs and also neglecting virtual pions. One might object that in our case the situation is not so clear because, even if it is true that the motion of quarks inside a particle is non-relativistic, the quarks find themselves in a very deep potential well, so deep as to cancel, practically, their rest energy, while this is not the case, of course, for nuclei. But this difference should not affect the conclusion stated above.

Consider in fact a very simple model of binding for quarks, a model which has only the purpose of illustrating the above point. Assume that quarks are bound through the intermediary of a neutral vector meson field, whose mass is chosen so as to give the requested long range (say $5 m_{\pi}$ as stated above) and whose coupling constant $g$ is also chosen so as to give the required depth for the potential well which binds the quarks.

Then, as is well known [7], the interaction of our quark system plus meson field can be transformed to all orders in $g$ so as to give rise to an instantaneous Yukawa interaction plus other terms, in a way entirely similar to that in which one obtains the Coulomb interaction in electrodynamics. In other words:

$$
\begin{aligned}
H_{\text {tranes }}^{\prime}= & \frac{g^{2}}{4 \pi} \int J_{0}(x) \frac{\exp -\mu\left|x-x^{\prime}\right|}{\left|x-x^{\prime}\right|} J_{0}\left(x^{\prime}\right) d^{3} x d^{3} x^{\prime}+ \\
& + \text { terms proportional to } \mathbf{J}(x) .
\end{aligned}
$$

Here $J_{0}(x)$ and $\mathbf{J}(x)$ are the time and the space components of the quark current

$$
J_{\mu}(x)=\sum_{1}^{3} \bar{\psi}_{i}(x) \gamma_{\mu} \psi_{i}(x)
$$

Now the conclusion stated above, that it is a good approximation to deal only with a fixed number of quarks, arises from the fact that the matrix elements of the kind $\left\langle q / H^{\prime} / q, \bar{q}, q\right\rangle$ are of the order $p / M$ where $p$ is the momentum of the initial or of one of the final quarks. If $p$ is the momentum of the initial quarks, this is small as we have said above. If it is the momentum of one of the final quarks this may be large, but then the transition becomes off the energy shell by an amount $M$ and is therefore improbable [8].

\section{Discussion of the Gell-Mann Okubo formula}

A second point which perhaps can be better understood, if the quarks move non-relativistically, is why the Gell-Mann Okubo [9] mass formula has a validity which appears to go much beyond first order perturbation theory.

Consider the baryon decuplet or octet and write an hamiltonian for a three quark system which consists of a unitary invariant part $H_{0}$ plus a part, $H_{1}$, which is assumed, as usual, to transform like the $T_{3}{ }^{3}$ component of a unitary tensor. $H_{0}$ will be e.g. of the form

$$
H_{0}=2 M+M^{\prime}+T_{1}+T_{2}+T_{3}+V(12)+V(13)+V(23)
$$

where $2 M+M^{\prime}$ is the rest energy of the quarks (two of them have the same mass $M$ and the third 
a different mass $M^{\prime}$ as discussed in more detail later); the $T_{i}{ }^{\prime} s$ are the kinetic energies of the quarks (with the same mass) [10] and the $V(i k)$ are the potential energies, written under the assumption of two body forces; this assumption is however irrelevant and all that has to be assumed of the hamiltonian $H_{0}(1)$ and of the potential energy in (1) is that it is a symmetric function of the quark coordinates and spins, independent of the unitary spin operators or containing them only through the Casimir invariants. On the other hand the part $H_{1}$ of the hamiltonian will be symmetric in the space, spin and unitary spin coordinates and have a linear dependence on the $\lambda_{i}{ }^{(8)}$ [11]. We can write

$$
H_{1}=H_{1}(T)+H_{1}(U)
$$

where $H_{1}(T)$ is the kinetic and rest-mass term contribution to the part of the hamiltonian transforming as $\lambda_{i}{ }^{(8)}$ and $H_{1}(U)$ is the potential energy contribution. For instance if there are only two body forces we can write for $H_{1}(U)$

$$
H_{1}(U)=U(12)\left(\lambda_{1}^{(8)}+\lambda_{2}^{(8)}\right)+U(13)\left(\lambda_{1}(8)+\lambda_{3}^{(8)}\right)+U(23)\left(\lambda_{2}(8)+\lambda_{3}^{(8)}\right)
$$

while $H_{1}(T)$ is of course given by:

$$
H_{1}(T)=\frac{1}{3}\left(M-M^{\prime}\right) \sum_{1}^{3} \lambda_{i}{ }^{(8)}+\sum_{1}^{3}\left(\frac{p_{i}{ }^{2}}{2 M}-\frac{p_{i}{ }^{2}}{2 M^{\prime}}\right) \lambda_{i}{ }^{(8)}
$$

where $M$ is the common mass of quarks of kind 1 and $2(p, n)$ and $M^{\prime}$ is the mass of the quarks of kind $3(\Lambda)$.

Let us now remark that the major contribution to the mass splitting both in the baryon decuplet and in the baryon octet may well arise from the first term in $H_{1}{ }^{(T)}$, that which describes the difference in mass between the third quark and the other two; that this mass difference can be responsible for the largest part of the splitting has been already pointed out by Zweig [4]. A measure of $M-M^{\prime}$ is given by the difference in mass $(M(\Sigma)+M(\Lambda) / 2)-M(n)=1154-938 \approx$ $216 \mathrm{MeV}$; or equivalently by the difference $M(\equiv)-(M(\Sigma)+M(\Lambda) / 2) \approx 175 \mathrm{MeV}$. That is we can take $M-M^{\prime}$ in the first term of (3) as having an order of magnitude around $200 \mathrm{MeV}$. To this strong perturbation, which, however, commutes with the remaining hamiltonian, and, therefore, can be treated exactly, a minor perturbation has to be added which is, for instance, responsible of the difference in mass between $\Lambda$ and $\Sigma ;(M(\Sigma)-M(\Lambda)) / 2$ is $\sim 40 \mathrm{MeV}$, so this should be the order of magnitude of this second perturbation. It is therefore not very strange that the first order calculation with this perturbation gives accurate results.

Now one would be tempted to identify this second perturbation with the second term of $H_{1}(T)$. Indeed if this were the case one would understand why also this second perturbation is linear in the $\lambda_{i}{ }^{(8)}$.

However the second (kinetic) term of (3) appears to be too small; it has in fact in order of magnitude $\left(M-M^{\prime}\right) \times<p^{2} / M^{2}>$ and for a non-relativistic motion of the quarks this cannot be much larger than $\sim 5$ or $10 \mathrm{MeV}$.

Therefore this " $40 \mathrm{MeV}$ " perturbation has probably to be found in the potential energy, although the question arises of why this perturbation should be simply linear in the $\lambda_{i}{ }^{(8)}$ [12]

In any case assuming the "40 $\mathrm{MeV} "$ perturbation to be due to a potential energy linear in the $\lambda_{i}{ }^{(8)}$ such as that given by equation (2), the effect of this perturbation on the masses of the baryons can be simply calculated in a way entirely similar to that in which one calculates the 
binding energies of the nuclei.

For instance the expectation value of $H_{1}(U)$ in the decuplet states can be immediately calculated as follows: call $X_{i}(i=1 \ldots 10)$ the 10 wave functions of the decuplet states. The $X_{i}$ are symmetric in the unitary spin variables and therefore can be written as the product of a completely antisymmetric space and spin part times the symmetric unitary spin functions which we shall call $W_{i}(i=1 \ldots 10)$. That is

$$
X_{i}=f(1,2,3) W_{i}
$$

where the antisymmetric $f(123)$ is the same for all the values of $i$. We therefore have

$$
\begin{aligned}
<\chi_{i}\left|H_{1}(U)\right|_{X_{i}}>=< & f(123) W_{i} \mid U(12)\left(\lambda_{1}(8)+\lambda_{2}(8)\right)+U(13)\left(\lambda_{1}(8)+\lambda_{3}(8)\right)+ \\
& +U(23)\left(\lambda_{2}(8)+\lambda_{3}(8)\right) \mid f(123) W_{i}>
\end{aligned}
$$

Now due to the factorized form of $X_{i}$ each of the addends in (4) separates into a space and spin matrix element and a unitary spin one. Due to the antisymmetry of $f(123)$ we have

$$
\begin{gathered}
\langle f(123)|U(12)| f(123)\rangle=\langle f(123)|U(13)| f(123)\rangle= \\
=<f(123)|U(23)| f(123)>=\bar{U} / 6
\end{gathered}
$$

Therefore, as expected

$$
<X_{i}\left|H_{1}(U)\right|_{X_{i}}>=\frac{\bar{U}}{3}<W_{i}\left|\lambda_{1}(8)+\lambda_{2}(8)+\lambda_{3}(8)\right| W_{i}>=\bar{U} Y_{i}
$$

where $Y_{i}$ is the hypercharge of the $i$-th decuplet particle. We can therefore write the mass formula for the decuplet as

$$
M_{i}=M_{0}+\left(M-M^{\prime}\right) Y_{i}+\bar{U} Y_{i}
$$

where, as we repeat, the dominant term proportional to $\left(M-M^{\prime}\right)$ is exact and only the last " $40 \mathrm{MeV} "$ term is to be regarded as calculated perturbatively (or variationally). A similar calculation of $H_{1}{ }^{(U)}$ can be performed of course for the baryon octet although there it is more complicated due to the fact that the space and spin part of the wave function is not antisymmetrical but transforms according to the 2 dimensional representation of the permutation group of three objects.

As far as the meson octets are concerned entirely similar considerations hold. Thinking of the mesons as a quark-antiquark compound we have of course to assume that there exist a strongly attractive unitary symmetric quark-antiquark force, perturbed again primarily by a perturbation due to the mass difference among quarks and, secondarily, by another perturbation linear in the $\lambda_{i}{ }^{(8)}$. Two remarks are appropriate here:

(1) It can appear strange that there are attractive forces both between quarks, and among quarks and antiquarks. For instance if the forces were only due to the neutral vector meson which was considered previously they would be attractive between quark and antiquark but repulsive between two quarks. Note however that the quark-quark force has a strength different from the quark-antiquark one. For instance assuming that the quark mass is $5 \mathrm{GeV}$, the binding between quark and an antiquark has an order of magnitude of $\sim 9.5 \mathrm{GeV}$, while the binding 
between two quarks has an order of magnitude of $(15-1) / 3 \sim 4.7 \mathrm{GeV}$. Therefore, roughly, the quark-antiquark binding is twice as strong as the quark-quark one. This might explain, by the way, the absence of particles built with two quarks; they would have a mass of the order of the quark mass. Of course the question remains of the non-existence of particles built with 4 or 5 quarks.

(2) In a scheme like the one presented here it is difficult to understand the fact that the formula for the meson masses is quadratic. This is not, however, typical of a non-relativistic approach like this. The explanations given so far for the fact that the meson mass formulas must be quadratic are indeed not convincing [13].

\section{Remarks on $\mathrm{SU}_{6}$}

If the internal structure of particles is non-relativistic, no particular difficulty arises in the problem of formulating $S U_{6}$ invariance. It is sufficient to assume that, in first approximation, our quark hamiltonian is independent of the spin; this requirement can be imposed easily on a non-relativistic system, as is well known. If we look at the $S U_{6}$ symmetry [14] from this point of view it is clear that $S U_{6}$ is an approximate symmetry violated possibly by nonrelativistic effects and certainly by relativistic ones; exactly as the wigner supermultiplet $\mathrm{SU}_{4}$ symmetry in nuclei is an approximate symmetry violated by tensor, spin orbit and spin-spin effects. The only problem becomes that of finding the kind of tensor, spin-orbit or spin-spin terms which do violate $S U_{6}$ symmetry. If the effects which these $S U_{6}$ violating terms produce in the wave function of a proton or of a neutron are not too large, $S U_{6}$ should be successful, while if the opposite is true $S_{6}$ should not be useful; which is the case is not yet entirely clear.

To consider a definite case we shall calculate [15] the ratio between the magnetic moment of proton and neutron by means of $S_{6}$ in the representation 56; this calculation becomes identical to the calculation of the ratio of the magnetic moments of $\mathrm{He}^{3}$ and $\mathrm{H}^{3}$ in a situation where:

(1) The space part of the wave function of these peculiar ${ }^{n} \mathrm{He}^{3 n}$ and ${ }^{n} \mathrm{H}^{3 n}$ nuclei built with quarks is antisymmetrical and has zero orbital angular momentum [16].

(2) The quarks have Bohr magnetic moments proportional to their charges, namely proportional to $2 / 3(e \hbar / 2 M c)$ for the $p$ quark, $-1 / 3(e \hbar / 2 M c)$ for the $n$ quark.

The calculation is straightforward. We call $p, n$, and $\Lambda$ the three quarks (of course $\Lambda$ does not intervene in this calculation) and note that the unitary spin part of the wave function for the proton and the neutron are, respectively

$$
{ }^{n} p^{n}=p_{1}\left(p_{2} n_{3}-p_{3} n_{2}\right)
$$

and

$$
n n=n_{1}\left(n_{2} p_{3}-n_{3} p_{2}\right)
$$

Calling $f(1,2,3)$ the space and spin part of the wave function, we now assume [16] that the space part of the wave function $X\left(r_{1}, r_{2}, r_{3}\right)$ is rotationally invariant $(L=0)$, depends only on the relative coordinates and is antisymetrical; that is we assume that it.is:

$$
f(123)=X\left(r_{1}, r_{2}, r_{3}\right) \alpha_{1}\left(\alpha_{2} \beta_{3}-\alpha_{3} \beta_{2}\right)
$$


where $X\left(r_{1}, r_{2}, r_{3}\right)$ is antisymmetrical and where the spin part is dictated by the requirement of having a spin $1 / 2$. This is the only point at which $S U_{6}$ intervenes; indeed, assuming that the octet and decuplet particles belong to the same 56 representation of $S U_{6}$ the space part of the wave function of all the decuplet and octet particles must be the same; but due to the Pauli principle, the space part of the decuplet, as we have already stated, must be antisymmetric, and this must be also the case for the octet space part; it seems appropriate to remark however that our assumption of antisymmetry of $X\left(r_{1}, r_{2}, r_{3}\right)$ is in fact weaker than to assume the validity of $S U_{6}$; it might well be that the shape of $X\left(r_{1}, r_{2}, r_{3}\right)$ for the octet particles is different from that for the decuplet particles (while $S U_{6}$ requires of course the same $X\left(r_{1}, r_{2}, r_{3}\right)$ for both) and still the results on the ratio between magnetic moments do hold provided that $X\left(r_{1}, r_{2}, r_{3}\right)$ is antisymmetric.

To proceed let us write the complete wave functions for proton and neutron

$$
\begin{aligned}
& ' p '=N \underline{\underline{A}} X\left(r_{1}, r_{2}, r_{3}\right) \alpha_{1}\left(\alpha_{2} \beta_{3}-\alpha_{3} \beta_{2}\right) p_{1}\left(p_{2} n_{3}-p_{3} n_{2}\right) \\
& " n "=N \underline{\underline{A}} X\left(r_{1}, r_{2}, r_{3}\right) \alpha_{1}\left(\alpha_{2} \beta_{3}-\alpha_{3} \beta_{2}\right) n_{1}\left(n_{2} p_{3}-n_{3} p_{2}\right)
\end{aligned}
$$

where $N$ is a normalization factor and $\underline{A}$ is an antisymmetrization operator. Because $X\left(r_{1}, r_{2}, r_{3}\right)$ is already antisymmetrical the spin and unitary spin part must be symmetrical. Assuming $X$ to be normalized we obtain:

$$
\begin{gathered}
" p^{n=} X\left(r_{1}, r_{2}, r_{3}\right) 1 / \sqrt{18}\left\{2\left[\alpha_{1} p_{1} \alpha_{2} p_{2} \beta_{3} n_{3}+\alpha_{1} p_{1} \alpha_{3} p_{3} \beta_{2} n_{2}+\alpha_{2} p_{2} \alpha_{3} p_{3} \beta_{1} n_{1}\right]-\right. \\
-\left[\alpha_{1} p_{1} \alpha_{2} n_{2} \beta_{3} p_{3}+\alpha_{1} p_{1} \beta_{2} p_{2} \alpha_{3} n_{3}+\alpha_{2} p_{2} \alpha_{1} n_{1} \beta_{3} p_{3}+\alpha_{2} p_{2} \beta_{1} p_{1} \alpha_{3} n_{3}+\right. \\
\left.\left.+\alpha_{3} p_{3} \alpha_{1} n_{1} \beta_{2} p_{2}+\alpha_{3} p_{3} \beta_{1} p_{1} \alpha_{2} n_{2}\right]\right\}
\end{gathered}
$$

and for $" n n$ a similar wave function where simply each $p$ is replaced by an $n$ and each $n$ with a $p$.

The expectation value of the spin magnetic moment operator (there is no orbital part because $L=0$ )

$$
\mu=\frac{e \hbar}{2 M c}\left(\frac{2}{3} a_{p}-\frac{1}{3} \sigma_{n}\right)
$$

(where $\sigma_{p}$ and $\sigma_{n}$ are the proton quark and neutron quark spins) is:

$$
\begin{gathered}
<\mu_{z}>_{n p n}=\frac{1}{18}\left[4 \times 3 \times\left(\frac{5}{3}\right)+6 \times\left(-\frac{1}{3}\right)\right] \frac{e \hbar}{2 M c}=\frac{e \hbar}{2 M c} \\
<\mu_{z}>_{n p "}=\frac{1}{18}\left[4 \times 3 \times\left(-\frac{4}{3}\right)+6 \times\left(\frac{2}{3}\right)\right] \frac{e \hbar}{2 M c}=-\frac{2}{3} \frac{e \hbar}{2 M c}
\end{gathered}
$$

One can see that the famous relation $[15]<\mu>_{p^{n}} /<\mu>_{n^{n}}=-3 / 2$ is obtained [17].

A few comments on the problem of reconciling $S U_{6}$ and Lorentz invariance, to which a great deal of attention has been devoted recently [18] and which indirectly stimulated the present paper appear appropriate at this point. It can first be said that in spite of the huge amount 
of work no satisfactory solution to this problem has appeared. Indeed it has not proven possible to construct a Lie group $G$ having as subgroups both the Lorentz group and the $S U_{6}$ group, without introducing at least 32 generators having an obscure physical meaning. Or stated differently, it does not appear possible, and in a sense it has been proven that is impossible [19], to construct a relativistic invariant Lagrangian for a system of interacting particles (containing only quantities having a physical meaning) which is also invariant under the transformations of the $\mathrm{SU}_{6}$ group.

Now one might ask: what is the relevance of the above conclusion for the point of view developed in the present paper? The answer is simply: no relevance at all. We don't require and we do not think that the quark Lagrangian is exactly invariant under a group $G$ such as that mentioned above. We simply require that the interactions are such that this $S U_{6}$ invariance possibly holds in the non-relativistic limit.

of course since reactions among particles, composed of quarks, take place also at relativistic velocities we must finally be able to construct a relativistic Lagrangian of interacting quarks; but we repeat: of this Lagrangian we should only require that be possibly invariant with respect to $S U_{6}$, in the non-relativistic limit, not that it is exactly $S U_{6}$ invariant.

If this is so a conjecture can be made: that $S U_{6}$ is possibly a good symmetry for non-relativistic phenomena but fails to be such when particles, and hence the quarks which they contain. collide at relativistic velocities [20].

To make an analogy let us fix our attention for a moment on a positronium atom. The energy levels of positronium are determined to a good accuracy by the Balmer formula. The spin orbit interaction or spin-spin interactions can be neglected to a good approximation. However if we want to treat reactions in which a fast positronium atom intervenes (consider for instance production of positronium by a high energy photon in the field of a nucleus) two things become necessary:

(1) A relativistic description of the positronium state; or in other words: how does the positronium wave function transform under a Lorentz transformation; this is a purely kinematic problem.

(2) A relativistic description of the interaction of the electrons in the positronium with other particles; in particular the spin orbit terms become important; this is a dynamical problem.

The problem of a particle composed of massive quarks is according to the point of view suggested in the present paper exactly similar to that of positronium; the problem is not that of constructing a Lagrangian which is simultaneously relativistically and $S U_{6}$ invariant. What apparently we have learnt instead is that in the non-relativistic limit the dynamics and the interactions become simple and this holds in particular, as we have seen, for the internal dynamics. Relativistically they presumably are more complicated; how much more complicated is a question which we must leave for the future.

\section{Final Remarks}

Three further remarks seem appropriate at this point.

(1) One might object that if the potential well representing the interaction among two quarks 
has a relatively long range, as assumed in this paper, the density of levels in this well might be too high as compared to the density of observed particles or resonances. This is not so, however; for instance, considering the mesons, taking a potential well with a range of $\left(5 m_{\pi}\right)^{-1}$ and assuming an infinite potential well the distance among the first two $S$ states is $27\left(5 m_{\pi}\right)^{2} / M \cong 20 m_{\pi}$ where the mass $M$ of a quark has been taken again to be $5 \mathrm{GeV}$. Of course there are also $p, d$, etc. levels, but on the whole the distance among two states does not seem too small, considering also the fact that in some states the forces may well be repulsive [2].

(2) An objection which might be raised against $S U_{6}$ or, more particularly, against the representation 56 for the baryons, is that it looks strange that the space part for the baryons is completely antisymmetrical; indeed the kinetic energy in antisymmetrical states is larger than in symmetrical states and it might appear strange that the baryon octet, which does constitute the ground state for three quarks, is a spatially antisymmetrical state. We have already expressed our point of view on the $\mathrm{SU}_{6}$ classification and noted that its validity (even non-relativistically) is not yet established. However this particular objection appears to us inconsistent because the problem here is entirely different from that in ordinary nuclear physics. Here the kinetic energy is only a very small perturbation with respect to the very large potential energy. Essentially what one has to minimize is the potential energy and it may well be that the forces holding together the quarks are of exchange character and attractive in spatially antisymmetric states.

(3) Finally, if the present ideas are valid, the quarks should exist; they should not be only mathematical entities. It has obviously no meaning to write an hamiltonian, that is an energy, for mathematical entities. So one should finally discover the quarks. Which are the most appropriate conditions for this should be investigated [22]. We hope to come back to this point in the future [23].

\section{References}

1. E. FERMI and C.N. YANG, Phys. Rev. 76, 1739 (1948).

2. M. GELL MANN, Physics Letters 8, 214 (1964).

3. T.D. LEE, F. GURSEY and M. NAUENBERG, Phys. Rev. 135, B467 (1964).

4. G. ZWEIG-CERN preprint $8419 /$ th 412 , Feb. 1964 .

5. M. GELL MANN, Phys. Rev. 125, 1067 (1962).

Y. NEEMAN, Nuclear Physics 26, 222 (1961).

6. For a recent survey of the situation compare A. SALAM: Proc. of the Dubna 1964 Conference on high energy physics (to be published).

7. Compare e.g. Y. FUJII, Progr. Theor. Physics 21, 232 (1959).

8. It must be pointed out, however, that a virtual $q \bar{q}$ system can be produced in a strongly bound state. In this case its mass is not of the order $M$ (the quark mass), but of the order of a few pion masses; the argument which we have just given should therefore not be taken 
as indicating for instance that a virtual process like $\pi \rightarrow \pi+\rho$ is weak or unimportant; in other words if we have a $q \bar{q}$ system representing a pion it certainly can be accompanied for part of the time by a $p$; or, if we have a $q q q$ system, say a nucleon, it certainly can be accompanied for part of the time by a $q \bar{q}^{\prime}$ system representing a pion. However in the same sense in which a rather accurate description of nuclei is possible without taking the exchange currents into account, that is in terms of the nucleon coordinates only, a description of the nucleons might be possible where only the quark coordinates intervene.

9. Compare ref. [5] and S. OKUBO, Progr. Theor. Physics 27, 949 (1962).

10. $T_{1}+T_{2}+T_{3}=\sum_{i}^{3} p_{i}{ }^{2} / 2 \mu ; \quad \mu=3 M M^{\prime} /\left(2 M^{\prime}+M\right)$.

11. The matrix $\lambda(8)$ will be taken, in this paper simply as $\left({ }^{1} 1_{-2}\right)$.

12. That this question is a real question, different from that of why once the perturbation is taken as being linear in the $\lambda_{i}{ }^{(8)}$ its second order effects are negligible, can be appreciated if we consider for a moment the problem of the Coulomb deviations from charge independence in ordinary nuclear physics. The terms which produce these deviations have the form

$$
\text { (*) } \frac{1}{2} \Sigma_{i}\left(M_{n}-M_{p}\right) \tau_{i}{ }^{(3)}+\Sigma_{i}\left(\frac{p_{i}^{2}}{2 M_{p}}-\frac{p_{i}{ }^{2}}{2 M_{n}}\right) \tau_{i}{ }^{(3)}+\sum_{i>J} \frac{e^{2}}{r_{i J}} \frac{\tau_{i}{ }^{(3)}+\tau_{J}{ }^{(3)}+\tau_{i}{ }^{(3)} \tau_{J}{ }^{(3)}}{4}
$$

The first two terms in $\left(^{*}\right)$ are the terms analogous to these of $H_{1}(T)$ in our case; in particular the second term is usually negligible just for the same reasons mentioned above; the third term in (*) (that arising from the Coulomb repulsion among protons) contains, as one can see, both terms linear in the $\tau_{i}{ }^{(3)}$ and terms bilinear in the $\tau_{i}(3)$ with the same strength. Why the terms quadratic of the kind $\lambda_{i}(8) \times \lambda_{J}{ }^{(8)}$ are absent in the "40 MeVn perturbation in our case or why, if present, they contribute practically nothing to the binding energies is the question which we have posed, but which we must leave unanswered in this paper.

13. OKUBO and RYAN (Nuovo Cimento 34, 776 (1964)) base their explanation on the fact that, if one writes $M^{2}=P_{\mu} P^{\mu}$ and decomposes $P_{\mu}$ as $P_{\mu}{ }^{(0)}+P_{\mu}{ }^{(8)}$ the terms of first order in $P_{\mu}(8)$ are quadratic in the masses; this argument appears to us inconsistent because it might be equally well be used to show that the mass formulas must be of order $2 n$ in the masses; one would have simply to write $M^{2 n}=\left(P_{\mu} P^{\mu}\right)^{n}$ and proceed in the same way as 0 kubo and Ryan do.

On the other hand the explanation given in reference (3) is based on the assumption of a zero order approximation in which all the bosons have zero mass.

14. F. GURSEY and L.A. RADICATI, Phys. Rev. Let ters 13, 173 (1964).

B. SAKITA, Phys. Rev. 136, B1756 (1964).

See also A. PAIS, Phys. Rev. Letters 13, 175 (1964).

15. M.A. BEG, B.W. LEE and A. PAIS, Phys. Rev. Letters 13, 514 (1964).

16. Contrary to what it might appear at first sight, there is nothing which prevents the construction of a wave function $X\left(r_{1}, r_{2}, r_{3}\right)$ totally antisymmetric with respect to $r_{1}, r_{2}, r_{3}$ depending only on the relative coordinates and rotationally invariant (that is with zero orbital angular momentum).

If we write $\mathbf{r}=\mathbf{r}_{1}-\mathbf{r}_{2}$ and $\rho_{=}=\mathbf{r}_{3}-\left(\mathbf{r}_{1}+\mathbf{r}_{2}\right) / 2$ the expression $X\left(r_{1}, r_{2}, r_{3}\right)=A(\mathbf{r} \cdot p \mid \mathbf{r}$ or the expression $A(\mathbf{r} \cdot \rho)|\mathbf{r}|^{4}$ where $A$ means antisymetrization with respect to $1,2,3$ is an example. 
Note however that for instance $A(\mathbf{r} \times \rho)$ or $A(\mathbf{r} \times p)|\mathbf{r}|^{2}$ do vanish identically. I am very grateful to Dr. C. Becchi for a remark on this point which has been essential for avoiding a mistake.

17. While the ratio $\mu_{p} / \mu_{n}$ is obtained correctly, the absolute values of the magnetic moments constitute a mystery. To obtain the correct absolute values one should assume that $e / M$ (where $M$ is the quark mass) is equal to $2.79 e_{/} M_{p}$ where $M_{p}$ is the proton mass; in other words the $g$ factor of the $p$ quark should be $(4 / 3) 2.79 M / M_{p}$; this is entirely mysterious.

Note also that, as far as the ratio among the magnetic moments is concerned, all values of the $p$ and $n$ quark charge in the ratio $2:-1$ lead to the $-3 / 2$ result; in other words, as far as this particular result is concerned the charges themselves need not be fractional. This observation may be of interest if two different triplets are assumed to exist with integral charge (compare H. BACRY, J.NUYTS and L.VAN HOVE, Physics Letters, 9, 279 (1964).

Note finally a third, more conventional, but nevertheless important and related question: can really the exchange currents (compare footnote (8)) be neglected in the calculation of the magnetic moments?

18. The paper by R. Delbourgo, A. SAlam and J. STRATHDeE (Proc. Roy. Soc. A284, 146 (1965)) contains much of the relevant bibliography; compare also W. RUHL, CERN preprints $10058 /$ Th 505 and $65 / 70 / 5 /$ Th 514 .

19. W.D. MCGLINN, Phys. Rev. Letters 12, 467 (1964).

W. RUHL, CERN preprint $9830 /$ Th 492 .

C. BECCHI (to be published).

20. If this is so one might take some two reactions capable of furnishing a test of $S U_{6}$ (we have not yet thought in detail which might be the most appropriate) and explore their behaviour with increasing energy. Assuming that at low energy (here low energy means low with respect to the masses of the intervening particles, not with respect to the mass of the quarks) the $S U_{6}$ predictions are satisfied, larger and larger deviations should occur with increasing energy. It should, however, be observed that at low energy the problem arises of the different $Q$ values or masses, so that it may be really difficult to find an energy region where the predictions of $\mathrm{SU}_{6}$ are satisfied.

21. Of course the excited states become more and more relativistic.

22. The present evidence (or better lack of evidence) for quarks has been discussed by M. SCHWARTZ in the Proceedings of the Galilec meeting in Pisa, September 1964 (to be published).

23. Note added in proof. (a) it may be of interest to point out that indirect tests of the present model are provided:

(1) by the radiative decay rates of vector mesons (C. BECCHI and G. MORPURGO, Phys. Rev., in course of publication);

(2) by the vanishing of the E2 amplitude in the $N_{33} *-N+\gamma$ transition (C. BECCHI and G. MORPURG0, Physics Letters, in course of publication).

(b) A preprint by $Y$. Nambu, received while the present paper was in the press (Dynamical symmetries and fundamental fields - EFINS-65-6), contains some ideas similar to those of the first section of the present paper. 